\section{Sauerstoff an Bord?}

Der Kabineninnendruck in Verkehrsflugzeugen wird abgesenkt, bis er etwa einer Höhe von 2.400 m über dem Meeresspiegel entspricht. Außerdem wird die Luft in der Kabine angewärmt, wodurch sie sehr trocken wird. Für gesunde Menschen ist das im Allgemeinen unproblematisch, da der verminderte Sauerstoffdruck durch eine schnellere, tiefere Atmung ausgeglichen wird. Patienten mit obstruktiven Atemwegserkrankungen können dagegen den verminderten Sauerstoffdruck unter Umständen nicht ausgleichen und sind daher besonders auf Langstreckenflügen auf eine zusätzliche Sauerstoffversorgung angewiesen. Etliche Fluggesellschaften untersagen jedoch die Mitnahme eines Sauerstoffvorrates in das Flugzeug. Betroffene müssen sich dann darauf verlassen, dass die Fluggesellschaft Sauerstoff zur Verfügung stellt.

Die Bedingungen für die Bereitstellung von Sauerstoff variieren erheblich zwischen den einzelnen Fluggesellschaften. Unter Umständen ist die Sauerstoffanwendung nur bei Start und Landung verboten. Gestattet eine Fluggesellschaft die Mitnahme von Gasdruckflaschen oder die Verwendung tragbarer Sauerstoffkonzentratoren in einem bestimmten Flugzeugmodell, so muss dies nicht automatisch für die ganze Flotte gelten.

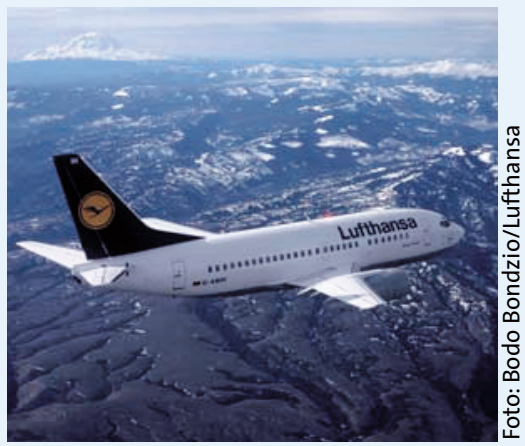

Es ist das Verdienst der European Lung Foundation akribisch alle europäischen Fluggesellschaften zu deren Bedingungen für eine zusätzliche Sauerstoffversorgung befragt und die Ergebnisse in einer Datenbank zusammengefasst zu haben. Diese Datenbank wurde jetzt von der Deutschen Atemwegsliga e.V. ins Deutsche übersetzt und ist ab sofort online unter www.de. european-lung-foundation.org/index. php?id=12409 abrufbar. Bei jeder Fluggesellschaft sind nicht nur die Informationen für die Sauerstoffmitnahme hinterlegt, sondern es ist auch eine Telefonnummer für Rückfragen angegeben.

\title{
MARC macht mobil gegen Allergien
}

Um Forschung und klinische Versorgung noch enger zu verzahnen, haben die Technische Universität München und das HelmholtzZentrum München im Dezember 2008 das Munich Allergy Research Center (MARC) aus der Taufe gehoben. Das Zentrum soll in Fragen von Allergie und Umwelt eine zentrale Anlaufstelle werden.

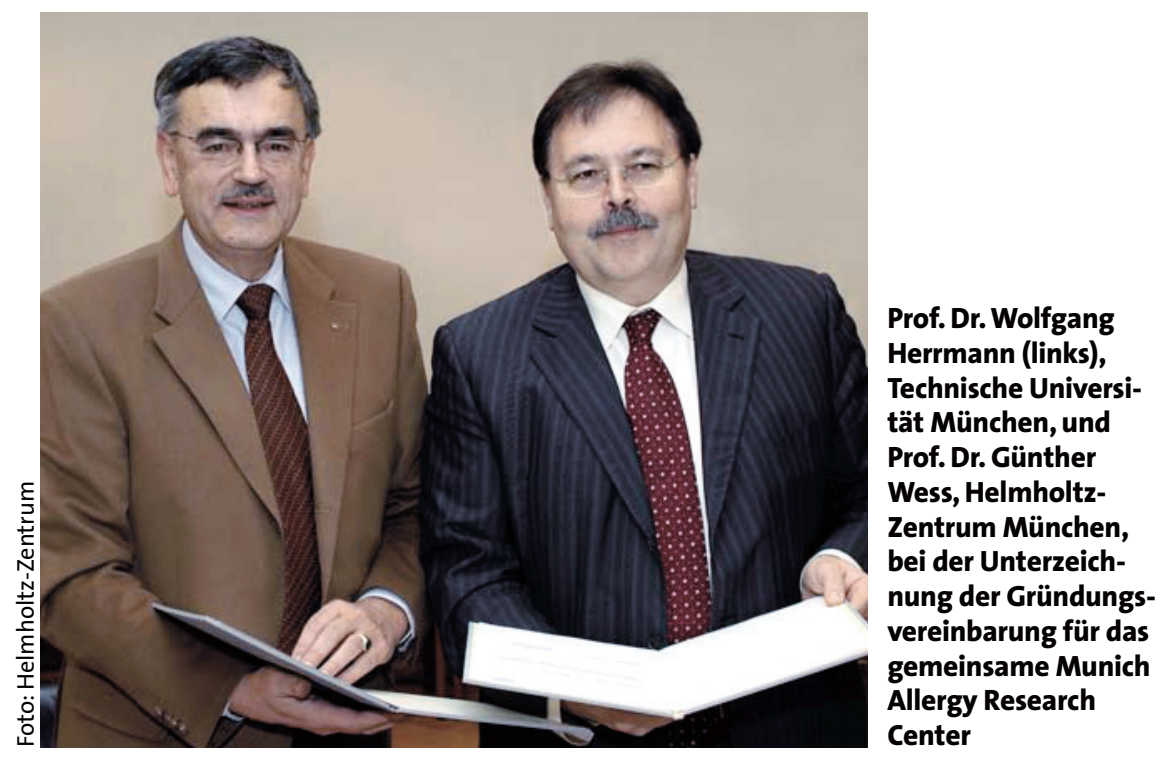

ern des MARC ist ein Lehrstuhl mit neuartiger Ausrichtung: Er verbindet in weltweit einmaliger Weise molekulare Allergologie und Umweltforschung. „Mit unserem Lehrstuhl für molekulare Allergologie und Umweltforschung schaffen wir eine Institution, die das bisherige Engagement von Helmholtz-Zentrum und Technischer Universität in der Allergieforschung nachhaltig fortsetzt," ist TU-Präsident Prof. Dr. Wolfgang Herrmann überzeugt. „Darüber hinaus ist das Research Center ein Beitrag zur deutschen Anpassungsstrategie an den Klimawandel."

Prof. Dr. Günther Wess, wissenschaftlich-technischer Geschäftsführer des Helmholtz-Zentrums München, hob bei der Gründungsfeier die Vorteile der interdisziplinären Vernetzung des MARC hervor: „Um das komplexe $\mathrm{Zu}$ sammenspiel von Umweltfaktoren und genetischer Veranlagung für Allergien verstehen zu lernen und um neue Ansät- ze für Therapie und Prävention zu entwickeln, müssen Mediziner und Naturwissenschaftler unterschiedlichster Fachrichtungen zusammenarbeiten. Insbesondere werden auch die Patienten von dieser neuen und engen Verbindung von Klinik und Grundlagenforschung direkt profitieren."

Insgesamt 20 Institute und Kliniken des Helmholtz-Zentrums München und der TU beteiligen sich am MARC. Der Inhaber des neuen Lehrstuhls für molekulare Allergologie und Umweltforschung wird auch das Zentrum Allergie und Umwelt an der TU München (ZAUM) leiten, das seit zehn Jahren besteht. „Netzwerke zu bilden ist in der Klima- und Allergieforschung essenziell“", betonte Herrmann weiter, „die Thematik ist so komplex und anspruchsvoll, dass sie auch von einer großen Universität alleine nicht geschultert werden kann. Das MARC ist daher offen für weitere Kooperationspartner." red 